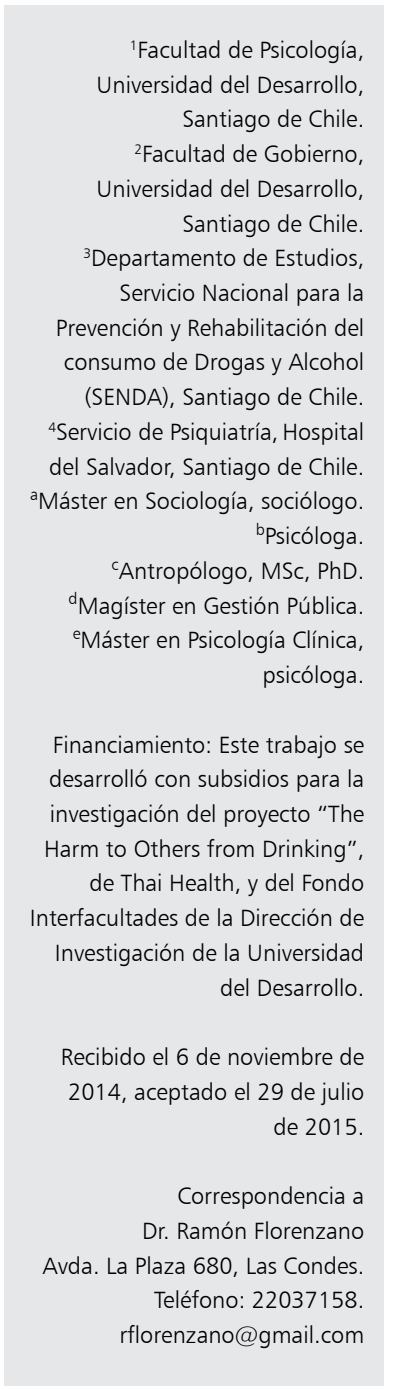

\section{Daño a terceros producido por el alcohol: resultados de un estudio poblacional en Chile}

\author{
RAMÓN FLORENZANO ${ }^{1,4}$, EUGENIO GUZMÁN ${ }^{2, a}$, \\ CATALINA SIEVERSON ${ }^{1, b}$, ÁLVARO CASTILLO-CARNIGLIA $^{3, c}$, \\ MIGUEL ÁNGEL FERNÁNDEZ ${ }^{2, \mathrm{~d}}$, ÁNGELA ECHEVERRÍA ${ }^{4, e}$, \\ MICHELLE BARR ${ }^{1, \mathrm{~b}}$
}

\section{The harm to others from drinking. A population study}

Background: Alcohol can harm both drinkers and their human environment. Most of research in Chile has focused on the drinker. This work focuses on the damage to others. Governmental expenditures due to drinking are estimated to quadruple the income earned through taxation. Aim: To report the results of a population survey conducted in Chile in 2013 to assess the harm done to others from drinking by a known or unknown drinker. Material and Methods: A household survey of a sample of 1500 subjects living in urban areas, predominantly over 50.000 inhabitants, was conducted by trained interviewers. Results: A significant number of respondents reported that the effect of alcohol consumption by third parties was somewhat (18.7\%) or very negative (14.8\%). The most frequent negative effects were verbal abuse (37.4\%), being bullied (17.4\%), feeling fearful at home or in a private meeting (19.6\%) and family problems (16.9\%). Conclusions: About one third of Chileans surveyed have suffered negative effects due to alcohol consumption of a known or unknown drinker. This finding signals the need of designing public policies addressed to minimize or prevent collateral effects of drinking.

(Rev Med Chile 2015; 143: 1242-1251)

Key words: Alcohol drinking; Alcohol induced disorders; Data collection.
$\mathrm{D}$ iversas organizaciones internacionales han subrayado la relevancia del estudio del daño a terceros que produce el consumo de alcohol. En el año 2008, la $61^{\circ}$ Asamblea Mundial de la Salud divulgó la "Estrategia para reducir el uso dañino del alcohol" , donde se señalaba que el consumo excesivo se asocia a diversos problemas sociales tales como delincuencia, violencia, desempleo y ausentismo laboral, generando altos costos sociales y de salud. La estrategia menciona también la necesidad de cuantificar mejor tales costos. Entre las políticas y medidas específicas recomendadas por la Organización Mundial de la Salud (OMS) para prevenir el consumo de alcohol y sus consecuencias, se encuentran aquellas centradas en disminuir la conducción de vehículos bajo la influencia del alcohol. Además, se ha recomendado focalizar los esfuerzos en la distribución final del alcohol y sus efectos en los barrios circundantes; en cambiar los entornos de vida nocturna donde se concentran los bebedores; y prevenir la violencia familiar (VIF) ligada al consumo abusivo dentro del hogar.

Un aspecto señalado por la OMS y la Organización Panamericana para la Salud (OPS), es la necesidad de mejorar la calidad de la información acerca del consumo de alcohol. El formato de los datos acerca del daño a terceros, pocas veces 
posibilita realizar comparaciones dentro o entre países ${ }^{2}$, lo que disminuye la calidad de las decisiones basadas en evidencia.

En el año 2010, se aprobó en Chile la "Estrategia Nacional sobre Alcohol" ${ }^{3}$ centrada en la reducción del consumo riesgoso de alcohol y sus consecuencias sanitarias y sociales, tanto para el consumidor como para quienes lo rodean. Las recomendaciones de esta estrategia han priorizado a los jóvenes y adolescentes en riesgo de convertirse en consumidores problemáticos, a las madres embarazadas y sus hijos con riesgo de desarrollar Síndrome Alcohólico Fetal, a la mortalidad por accidentes de tránsito debido al consumo, al costo social asociados a VIF y criminalidad y las consecuencias laborales asociadas.

Siendo Chile un país vitivinícola, la producción de alcohol ha sido una fuente importante de ingresos, de oferta/demanda laboral y de impuestos. Esto contrasta con la preocupación histórica por medir el consumo de alcohol ${ }^{4}$ y estimar lo daños y costos individuales y sociales ${ }^{5}$ asociados al beber excesivo. En relación a otros países del mundo, Chile tiene un nivel intermedio de consumo de alcohol per cápita (8,2 litros de alcohol puro por cada persona mayor de 15 años) $)^{6}$. Sin embargo, además del volumen de consumo global, existen bebedores con un patrón de consumo riesgoso, caracterizado principalmente por la ingesta de altas cantidades de alcohol en períodos cortos de tiempo, cuyo resultado es una intoxicación por alcohol o la embriaguez. Este consumo se asocia a conductas riesgosas con importantes consecuencias para la salud y la sociedad, entre ellas manejar en estados de ebriedad, involucrarse en riñas, conducta sexual desprotegidas, riesgos durante el embarazo y ausentismo laboral.

La información acerca del daño a terceros ("Harm to Others"-H2O) en Chile es limitada. Los datos existentes no se recolectan sistemáticamente, lo que dificulta desarrollar medidas preventivas adecuadas a nivel local y nacional. Se ha estimado el costo económico del alcoholismo en $1979^{7}$ y en 2010 por el Ministerio de Salud de Chile (MINSAL) ${ }^{8}$, quien informa que el gasto gubernamental total fue de US\$210, per cápita por año ${ }^{9}$, incluyendo costos indirectos (pérdida de productividad, muertes prematuras, y ausentismo laboral) y directos (salud, seguridad ciudadana, accidentes del tránsito, etc.). Se han estimado también los beneficios económicos: US\$59 per cápita por año (exportaciones, fuerza laboral e impuestos). El daño a terceros más publicitado ha sido por accidentes de tránsito: entre 2000 y 2011 el alcohol estuvo involucrado en $8,28 \%$ del número total de accidentes, ocupando el segundo lugar en términos de mortalidad (19,9\%), y el cuarto en lesiones por accidente de tránsito $(10,3 \% \text { del total })^{10}$.

La relación entre consumo de alcohol y violencia intrafamiliar (VIF), incluyendo el abuso sexual, ha sido estudiada en Norteamérica, donde la disponibilidad física de alcohol se relaciona con un incremento de la violencia interpersonal y familiar $^{11}$, y en Chile, donde UNICEF $^{12}$ reporta en 2006 que cuando uno o ambos padres se embriagan frecuentemente, ejercen más violencia sobre sus hijos que cuando los padres nunca beben. La VIF es un factor de riesgo para que la víctima presente problemas psiquiátricos a mediano y largo plazo: un estudio chileno ${ }^{13}$ ha encontrado que el maltrato infantil se correlaciona con la presencia de trastorno de estrés postraumático en el adulto.

Estudios internacionales han documentado la relación entre consumo de alcohol por los padres, especialmente el padre, disfunción familiar y riesgo suicida a lo largo del ciclo vital. Un estudio estadounidense realizado con una cohorte retrospectiva de 17.337 mujeres adultas ${ }^{14}$, encontró que el alcoholismo, los trastornos afectivos y el uso de drogas por parte de los padres predecía psicopatología y suicidalidad en la adultez.

En un análisis casuístico realizado en un hospital general en Chile ${ }^{15}$, se revisó el antecedente de uso de alcohol entre parientes cercanos de pacientes adultos. De 7.476 casos atendidos entre 2004 y 2013, 11,8\% señaló que un pariente cercano bebía en exceso o era un alcohólico. Estos porcentajes aumentaron a $42,9 \%$ en aquellos tratados por farmacodependencias, a $21 \%$ de trastornos de personalidad y a 20,3\% de los trastornos afectivos. En ese mismo estudio, se constató que la mayoría de los parientes consumidores fueron los padres, y luego sus madres, abuelos o hermanos.

La relación entre consumo de alcohol y violencia comunitaria ha sido estudiada en Chile por el Servicio Nacional de Prevención de Drogas y Alcohol (SENDA) ${ }^{16}$. En una muestra de 16.000 adultos chilenos, representativos de la población general, 7,4\% de los entrevistados declara haber sido asaltado por alguien bajo la influencia del alcohol, así como 1,4\% declaró haber sido sujeto 
de una agresión sexual. Los asaltos fueron más frecuentes entre los hombres, y el abuso entre las mujeres. Otro estudio chileno ${ }^{17}$ analizó en 2013 la asociación entre consumo riesgoso de alcohol e inseguridad del barrio de residencia, observándose un riesgo elevado en aquellas personas que reconocen vivir en barrios percibidos como inseguros.

El objetivo de este trabajo es describir los resultados en Chile de una investigación que mide y analiza el daño a terceros producido por la ingesta de alcohol en países de ingresos bajos y medio-bajos, dentro de un estudio colaborativo internacional que está siendo aplicado en seis países $^{18}$. El objetivo específico fue comparar la percepción del efecto del beber en terceros afectados en forma positiva, neutra o negativa, analizando la variación de esta percepción según características socio-demográficas, relación con el bebedor, y medidas de bienestar subjetivo.

\section{Metodología}

Este es un estudio descriptivo transversal, realizado en el marco del proyecto colaborativo de Thai Health y OMS: "The Harm of Others From Drinking: A WHO/Thai International Research Project", aprobado por el Comité de Ética Institucional de la Universidad del Desarrollo y por el Ethical Review Committee de la OMS.

El muestreo se realizó considerando el estándar usado en el proyecto multinacional WHO/Thai Health, estableciendo como criterio encuestar a personas mayores de 18 años, con capacidad y/o disposición para responder a una encuesta que dura aproximadamente $45 \mathrm{~min}$. La agencia que habitualmente realiza estudios para la Facultad de Gobierno de la Universidad del Desarrollo (CIS-CHILE) seleccionó a los sujetos mediante muestreo probabilístico multi-estratificado por comuna, manzana y vivienda. Para ello se utilizó un mapa de cada comuna, en un universo de 12.291.000 de acuerdo a la base CASEN 2011. la unidad de muestreo fueron las viviendas. Para ello se elaboró un listado de las viviendas, y mediante un generador de números aleatorios se escogieron las viviendas a ser encuestadas. En los casos donde no había habitante en el domicilio, o bien no hubo disposición a participar en el estudio, o la persona no respondió por completo la encuesta, se reemplazó por la vivienda más cercana. La muestra fue sin cuota de sexo, ocupación u otra variable. La muestra final encuestada fue de 1.500 personas y el margen de error absoluto fue de 2,5\%, con un 95\% de confianza. Para llegar a esos 1.500 casos válidos, se entrevistaron 2.089 personas, por lo que la tasa de respuesta real fue de $71,8 \%$.

La muestra se concentró predominantemente en poblaciones urbanas superiores a 50.000 habitantes, debido a que encuestar en sectores rurales de difícil acceso superaba el presupuesto asignado al país por las agencias financiadoras. Sin embargo, la muestra de este estudio representa a 12.291.000 personas, es decir a $87 \%$ de la población de acuerdo al Instituto Nacional de Estadísticas para $2013^{19}$. La composición detallada de la muestra y las preguntas utilizadas se encuentran disponibles solicitándolas al autor principal.

El cuestionario fue traducido al español a partir la versión original en inglés, diseñada por el equipo del proyecto colaborativo18, y re traducido al inglés para evaluar la consistencia entre ambas versiones, siguiendo un protocolo de la $\mathrm{OMS}^{20}$. El instrumento aplicado mide los efectos del beber de otros a nivel personal, familiar, social y económico, la relación entre el bebedor y el encuestado e incluye 11 grupos de preguntas: 22 sobre características socio-demograficas y del hogar, 6 sobre bienestar subjetivo y estado de salud, 13 sobre percepción ocasionado al encuestado por el uso de alcohol de terceros, seis sobre las características de los bebedores excesivos mencionados por el encuestado, cuatro sobre la atención prestada a los bebedores por el encuestado, cuatro sobre las características socio-demográficas de los encuestados, siete sobre los efectos del bebedor sobre los niños, cuatro sobre los efectos laborales, dos sobre los efectos en el barrio, seis sobre las conductas de búsqueda de ayuda para el bebedor, y cinco sobre las características del consumo del encuestado (frecuencia, cantidad, puntaje del efecto. Un análisis de confiabilidad interescala mostró una consistencia interna con un Alfa de Cronbach de 0,807 .

Los entrevistadores fueron capacitados por el equipo de investigadores del proyecto, quienes a su vez fueron entrenados por videoconferencia por los investigadores principales del estudio internacional.

El instrumento se aplicó en los hogares de los entrevistados entre mayo y julio de 2013, con una duración de 45 min en promedio. Las respuestas fueron registradas directamente en un 
computador portátil, lo que permitió validación automática de calidad de las respuestas. El análisis cuantitativo fue realizado con el software PABTSSPSS en su versión 20.0, realizándose estadísticas descriptivas, y cálculo de diferencias paramétricas o no paramétricas entre los grupos comparados.

\section{Resultados}

La Tabla 1 exhibe las características socio-demográficas comparativas según la percepción por el efecto del consumo de alcohol por terceros, comparando aquellos que perciben el efecto como

Tabla 1. Características socio-demográficas y percepción del efecto general§ del consumo de alcohol por terceras personas, en los últimos 12 meses. Encuesta poblacional en Chile, 2013

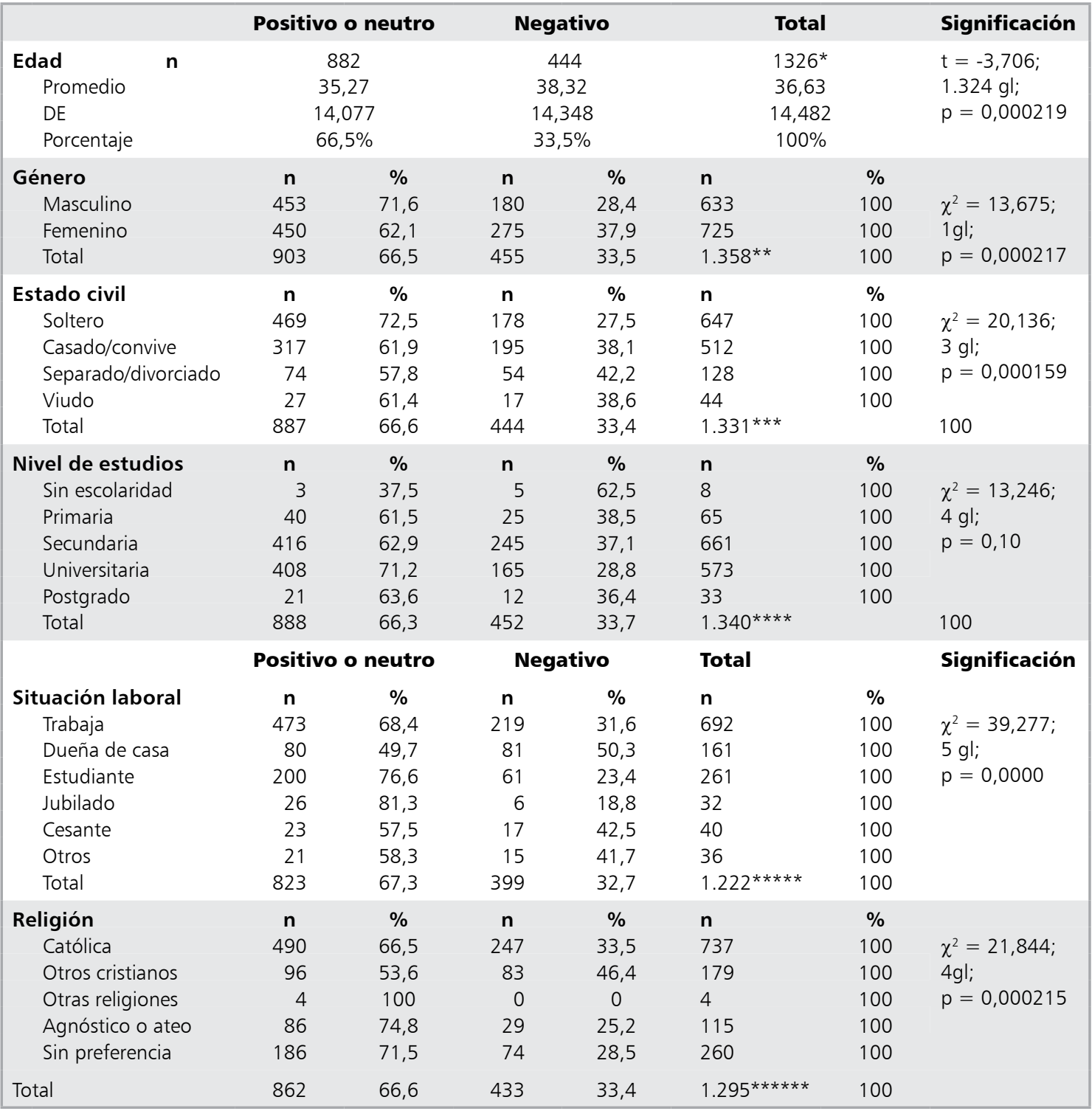

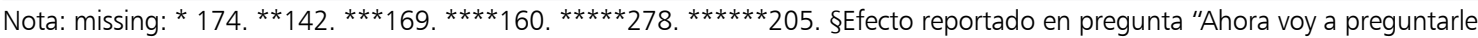
sobre el efecto general que tiene el consumo de alcohol de otras personas en su vida, durante los últimos 12 meses. ¿Diría usted que el efecto general ha sido muy positivo, algo positivo, neutral, algo negativo o muy negativo?". DE = desviación estándar. 
"positivo o neutro" con los aquellos que lo perciben como "negativo". Informan significativamente más efectos "negativos" los sujetos de mayor edad (38,32 años), las mujeres y los casados. En el nivel de educación se aprecia una gradiente de mayor efecto negativo percibido por el grupo de menor educación $(62,5 \%)$ hasta el universitario $(28,8 \%)$. En cuanto a la situación laboral también se observan diferencias estadísticamente significativas: las dueñas de casa fueron las que informaron de más efectos adversos de bebedores en su entorno $(50,3 \%)$, seguidos por los cesantes $(42,5 \%)$ y quienes trabajaban $(31,6 \%)$. Los estudiantes y jubilados fueron quienes reportaron menos efectos negativos ( $23,4 \%$ y $18,8 \%$, respectivamente). En cuanto a afiliación religiosa, los cristianos reformados (que en la muestra eran preferentemente evangélicos) fueron más negativamente afectados $(46,4 \%)$ que los pertenecientes a otras religiones, como la católica $(33,5 \%)$ o que los no creyentes $(25,2 \%)$. Estas diferencias también alcanzaron significación estadística.

La Tabla 2 muestra que 33,5\% de quienes respondieron indican que el efecto es algo o muy negativo, afectando especialmente al grupo de 45 a 64 años (43,5\%).

Como se aprecia en la Tabla 3, el efecto es percibido como negativo especialmente cuando los bebedores son miembros del hogar $(43,8 \%)$, seguidos por amigos $(28,4 \%)$, individuos cercanos $(17,6 \%)$ y compañeros de trabajo $(10,2 \%)$.

La Tabla 4 detalla los efectos negativos percibidos: $37,4 \%$ señalan que han sufrido maltratos verbales o insultos, seguido por agresión física menor (ser empujado) $(21,3 \%)$. Se sintieron atemorizados en lugares públicos en $19,6 \%$, o acosados en fiestas o lugares privados $16,3 \%$. Problemas familiares o matrimoniales se vieron afectados

Tabla 2. Comparación por edad del efecto del consumo de alcohol de terceros en los últimos 12 meses. Encuesta poblacional en Chile, 2013

\begin{tabular}{|c|c|c|c|c|c|c|c|c|c|c|c|c|c|}
\hline \multirow{2}{*}{$\begin{array}{c}\begin{array}{c}\text { Tramo } \\
\text { de edad }\end{array} \\
18-29\end{array}$} & \multicolumn{2}{|c|}{$\begin{array}{c}\text { Muy } \\
\text { positivo }\end{array}$} & \multicolumn{2}{|c|}{$\begin{array}{c}\text { Algo } \\
\text { positivo }\end{array}$} & \multicolumn{2}{|c|}{ Neutral } & \multicolumn{2}{|c|}{$\begin{array}{c}\text { Algo } \\
\text { negativo }\end{array}$} & \multicolumn{2}{|c|}{$\begin{array}{c}\text { Muy } \\
\text { negativo }\end{array}$} & \multicolumn{2}{|c|}{ Total } & \multirow{4}{*}{$\begin{array}{l}\text { Significación } \\
\chi^{2}=28,788 ; \\
12 \mathrm{gl} \\
p=0,004\end{array}$} \\
\hline & 22 & $4,0 \%$ & 56 & $10,2 \%$ & 308 & $56,2 \%$ & 91 & $16,6 \%$ & 71 & $13 \%$ & 548 & $100 \%$ & \\
\hline $30-44$ & 17 & $4,0 \%$ & 33 & $7,7 \%$ & 244 & $56,7 \%$ & 85 & $19,8 \%$ & 51 & $11,9 \%$ & 430 & $100 \%$ & \\
\hline $45-64$ & 11 & $3,8 \%$ & 12 & $4,1 \%$ & 142 & $48,6 \%$ & 63 & $21,6 \%$ & 64 & $21,9 \%$ & 292 & $100 \%$ & \\
\hline 65 y más & 2 & $3,6 \%$ & 5 & $8,9 \%$ & 30 & $53,6 \%$ & 9 & $16,1 \%$ & 10 & $17,9 \%$ & & $100 \%$ & \\
\hline Total & 52 & $3,9 \%$ & 106 & $8,0 \%$ & 724 & $54,6 \%$ & 248 & $18,7 \%$ & 196 & $14,8 \%$ & 1326 & $100 \%$ & \\
\hline
\end{tabular}

Tabla 3. Comparación por relación con el bebedor*, cuyo consumo tuvo un efecto negativo más significativo en el encuestado y percepción general del consumo de alcohol por terceras personas. Encuesta poblacional en Chile, 2013

\begin{tabular}{|lccccccc|}
\hline & \multicolumn{4}{c}{ Percepción general } & \multicolumn{2}{c|}{ Total } & Significación \\
\hline Relación** & Positivo o neutro & \multicolumn{2}{c}{ Negativo } & \multicolumn{2}{c|}{ Tot } \\
\hline Miembro del hogar & 65 & $27,4 \%$ & 77 & $43,8 \%$ & 142 & $34,4 \%$ & $\chi^{2}=13,185 ;$ \\
\hline Otros individuos cercanos & 43 & $18,1 \%$ & 31 & $17,6 \%$ & 74 & $17,9 \%$ & $3 \mathrm{gl} ;$ \\
\hline Amigo & 97 & $40,9 \%$ & 50 & $28,4 \%$ & 147 & $35,6 \%$ & 0,004 \\
\hline Compañero de trabajo/vecino & 32 & $3,5 \%$ & 18 & $10,2 \%$ & 50 & $12,1 \%$ \\
\hline Total & 237 & $100 \%$ & 176 & $100 \%$ & $413^{\S}$ & $100 \%$ \\
\hline
\end{tabular}

*Bebedor regular de alcohol que consume al menos una vez por semana. **Basada en la pregunta: "Pensando en todas las personas en su vida que usted ha mencionado anteriormente, aquellos cuyo consumo le ha afectado negativamente, en general, ¿cuál de esas personas diría Ud. que lo ha afectado más negativamente en los últimos 12 meses a causa de su consumo?". §Número de encuestados que reportaron algún problema con bebedores regulares de alcohol. 
Tabla 4. Comparación por percepción general del consumo de alcohol por terceras personas y problemas ocasionados (situaciones múltiples). Encuesta poblacional en Chile, 2013

\begin{tabular}{|c|c|c|c|c|c|c|c|}
\hline \multirow[b]{2}{*}{ Insultado o maltrato verbal } & \multicolumn{2}{|c|}{$\begin{array}{c}\text { Positivo o } \\
\text { neutro }\end{array}$} & \multicolumn{2}{|c|}{ Negativo } & \multicolumn{2}{|c|}{ Total } & \multirow{2}{*}{\begin{tabular}{c}
\multicolumn{2}{c}{ Significación } \\
$\mathbf{1}$ gl p* \\
0,000
\end{tabular}} \\
\hline & 212 & $23,5 \%$ & 170 & $37,4 \%$ & 382 & $28,1 \%$ & \\
\hline Empujones & 136 & $15,1 \%$ & 97 & $21,3 \%$ & 233 & $17,2 \%$ & 0,004 \\
\hline Daño físico & 49 & $5,4 \%$ & 42 & $9,2 \%$ & 91 & $26,7 \%$ & 0,008 \\
\hline Daño de ropa u otras pertenencias & 49 & $5,4 \%$ & 25 & $5,5 \%$ & 74 & $5,4 \%$ & 0,958 \\
\hline Accidente de tránsito & 33 & $3,7 \%$ & 15 & $3,3 \%$ & 48 & $3,5 \%$ & 0,736 \\
\hline Daño a casa, auto o propiedad & 58 & $6,4 \%$ & 44 & $9,7 \%$ & 102 & $7,5 \%$ & 0,032 \\
\hline Pasajero en un vehículo conducido por persona ebria & 172 & $19 \%$ & 72 & $15,8 \%$ & 244 & $18 \%$ & 0,144 \\
\hline Acoso en fiesta o lugar privado & 162 & $17,9 \%$ & 74 & $16,3 \%$ & 236 & $17,4 \%$ & 0,442 \\
\hline Sensación de amenaza o miedo en casa u otro lugar privado & 102 & $11,3 \%$ & 89 & $19,6 \%$ & 191 & $14,1 \%$ & 0,00004 \\
\hline Problemas familiares o dificultades matrimoniales & 82 & $9,1 \%$ & 77 & $16,9 \%$ & 159 & $11,7 \%$ & 0,00002 \\
\hline Problemas amigo o vecino & 106 & $11,7 \%$ & 63 & $13,8 \%$ & 169 & $12,4 \%$ & 0,267 \\
\hline Problemas financieros & 37 & $4,1 \%$ & 38 & $8,4 \%$ & 75 & $5,5 \%$ & 0,001198 \\
\hline
\end{tabular}

*Prueba de $\chi^{2}$.

negativamente en $16,9 \%$ y $8,4 \%$ enfrentaron a problemas financieros como consecuencia de la ingesta de alcohol de terceros. El verse involucrado en un accidente en vehículo por conductor ebrio fue menos reportado $(3,3 \%)$ que los anteriores.
Como se aprecia en la Tabla 5, el efecto más frecuente del consumo de terceros percibido por los encuestados fue el daño al entorno social $(28 \%)$, fallas en compromisos del bebedor $(23,9 \%)$, seguido por dejar de relacionarse con este

Tabla 5. Comparación por percepción general del consumo de alcohol por terceras personas y problemas ocasionados en el círculo cercano de personas, en los últimos 12 meses (respuestas múltiples). Encuesta poblacional en Chile, 2013

\begin{tabular}{|c|c|c|c|c|c|c|c|}
\hline \multirow[b]{2}{*}{ Emocionalmente herido } & \multicolumn{2}{|c|}{$\begin{array}{c}\text { Positivo o } \\
\text { neutro }\end{array}$} & \multicolumn{2}{|c|}{ Negativo } & \multicolumn{2}{|c|}{ Total } & \multirow{2}{*}{\begin{tabular}{c}
\multicolumn{2}{c}{ Significación } \\
$\mathbf{1}$ gl p* \\
0,00001
\end{tabular}} \\
\hline & 74 & $8,6 \%$ & 73 & $16,9 \%$ & 147 & $11,4 \%$ & \\
\hline Individuo dejó de relacionarse con personas por el bebedor & 102 & $11,9 \%$ & 85 & $19,5 \%$ & 187 & $14,5 \%$ & 0,00021 \\
\hline Forzada/o a tener relaciones sexuales & 28 & $3,3 \%$ & 10 & $2,3 \%$ & 38 & $3 \%$ & 0,333 \\
\hline Daño en entornos sociales & 166 & $19,4 \%$ & 121 & $28 \%$ & 287 & $22,3 \%$ & 0,00047 \\
\hline Bebedor falló en compromisos pactados & 136 & $15,8 \%$ & 104 & $23,9 \%$ & 240 & $18,5 \%$ & 0,000385 \\
\hline Bebedor falló en labores del hogar & 35 & $4,1 \%$ & 59 & $13,6 \%$ & 94 & $7,3 \%$ & 0,000000 \\
\hline Bebedor generó vergüenza social frente a amigos & 39 & $4,5 \%$ & 52 & $12 \%$ & 91 & $7,1 \%$ & 0,000001 \\
\hline Falta de alimentos en el hogar del bebedor & 13 & $1,5 \%$ & 14 & $3,2 \%$ & 27 & $2,1 \%$ & 0,042 \\
\hline Individuo debió marcharse por conductas del bebedor & 31 & $3,6 \%$ & 24 & $5,6 \%$ & 55 & $4,3 \%$ & 0,102 \\
\hline Perjuicio económico al hogar & 12 & $1,4 \%$ & 42 & $9,7 \%$ & 54 & $4,2 \%$ & 0,00000 \\
\hline Robo de objetos de valor & 15 & $1,8 \%$ & 23 & $5,3 \%$ & 38 & $3,0 \%$ & 0,00032 \\
\hline
\end{tabular}

*Prueba de $\chi^{2}$. 


\begin{abstract}
Tabla 6. Comparación por percepción general del consumo de alcohol por terceras personas y problemas ocasionados por desconocidos, en los últimos 12 meses (respuestas múltiples). Encuesta poblacional en Chile, 2013
\end{abstract}

\begin{tabular}{|c|c|c|c|c|c|c|}
\hline \multirow{3}{*}{ Acosado o ser molestado en la calle o en otro lugar público } & \multirow{2}{*}{$\begin{array}{l}\text { Positivo o } \\
\text { neutro }\end{array}$} & \multirow{2}{*}{\multicolumn{2}{|c|}{ Negativo }} & \multirow{2}{*}{\multicolumn{2}{|c|}{ Total }} & Significación \\
\hline & & & & & & $1 \mathbf{g l}$ \\
\hline & $27431,3 \%$ & 151 & $33,9 \%$ & 425 & $32,2 \%$ & 0,350 \\
\hline Sentir miedo al encontrarse al bebedor en la calle & $195 \quad 22,3 \%$ & 121 & $27,9 \%$ & 316 & $24,2 \%$ & 0,027 \\
\hline $\begin{array}{l}\text { Desvelo nocturno debido a ruidos molestos a causa de un } \\
\text { borracho }\end{array}$ & $270 \quad 30,5 \%$ & 182 & $40,7 \%$ & 452 & $33,9 \%$ & 0,0002 \\
\hline Inseguridad en algún lugar público & $23927,1 \%$ & 154 & $35,0 \%$ & 393 & $29,7 \%$ & 0,003 \\
\hline Ser incomodado/ molestado por bebedores desconocidos & $30634,5 \%$ & 205 & $45,9 \%$ & 511 & $38,3 \%$ & 0,00005 \\
\hline
\end{tabular}

*Prueba de $\chi^{2}$.

mismo (19,5\%) y sentirse emocionalmente herido $(16,9 \%)$. En porcentajes inferiores se constató el ser forzada/o a tener relaciones sexuales, falta de alimentos en el hogar y robos de objetos de valor.

En la Tabla 6, el efecto negativo más común causado por terceros desconocidos son la exposición a ruidos molestos (40,7\%), seguida por la inseguridad en un lugar público (35\%), ser molestado o acosado (33,9\%). La situación menos frecuente es el miedo al encuentro con un sujeto bajo la influencia del alcohol en la vía pública $(27,9 \%)$.

\section{Discusión}

Este trabajo es una primera aproximación descriptiva a un tema poco estudiado en nuestro medio. Su hallazgo central es que una proporción apreciable (más de 30\%) de los encuestados informó haber experimentado un efecto adverso debido al beber de terceras personas en los últimos doce meses. Asimismo, los efectos negativos son percibidos de manera más frecuente entre aquellos sujetos que poseen una mayor cercanía al individuo afectado (miembros del hogar y personas cercanas). Es de interés que el efecto del alcohol fue percibido como neutro por un porcentaje mayoritario $(54,6 \%)$ de los encuestados, como se ve en la Tabla 2. Este hallazgo muestra que el alcohol no afecta positiva ni negativamente a más de la mitad de la población encuestada, como el trabajo se centra en detallar los efectos negativos, las tablas restantes se centran en ese grupo, y se colapsaron las categorías positivo y neutro en un grupo de comparación,

Un alto porcentaje de individuos reportan que el consumo de alcohol de terceros ha tenido efectos neutros (aproximadamente 50\% de la muestra) y un porcentaje menor $(11,9 \%)$, indicó efecto positivo en el último año. El consumo de alcohol no es por lo tanto un elemento motivador central para casi la mitad de los encuestados, y sus efectos positivos son apreciados como tales por algo más de un décimo de ellos. La conexión promovida por la industria que liga el consumo a la alegría y la fiesta, no es la percepción mayoritaria de quienes rodean a los bebedores. En esa misma Tabla, se puede ver que la percepción es más negativa con respecto al consumo por familiares (\%) que con respecto a los amigos (\%) o colaboradores laborales (\%). La cercanía de la convivencia puede explicar el aplicar juicios diversos con respecto al efecto del consumo de alcohol,

El tipo de daño más publicitado en los medios (el efecto en los accidentes de tránsito producidos por bebedores), se produce en forma poco frecuente en la vida habitual de los encuestado, por lo que la percepción negativa es baja $(3,7 \%)$ si se le compara con los daños relacionados a insultos o situaciones molestas de menores consecuencias, Esto puede relacionarse a que el daño objetivo, material (como el producido por un accidente del tránsito) es menor que el subjetivo: el temor al subirse a un vehículo conducido por un bebedor ebrio, o a ser empujado en la calle a la salida de un lugar de expendio de alcohol. Las políticas públicas y legislación reciente se dirigen al daño objetivo, si 
bien infrecuente, muy significativo para el individuo y la sociedad. El mismo comentario se aplica a la percepción del abuso sexual que es infrecuente pero tiene consecuencias materiales y psicológicas importantes,

La caracterización sociodemográfica muestra que son las mujeres, dueñas de casa y los cristianos reformados, quienes perciben en mayor magnitud los efectos negativos producto del consumo de alcohol de terceros.

Los datos analizados corresponden a la realidad percibida por los adultos encuestados. En posteriores publicaciones se compararán los datos chilenos con los de otros países que utilizaron la misma encuesta y metodología de muestreo. Mientras tanto, la comparación con investigaciones semejantes publicadas en Estados Unidos de Norteamérica ${ }^{21}$ y Nueva Zelanda ${ }^{21}$ obtienen resultados similares en cuanto a porcentaje de efectos negativos. También en los estudios australianos ${ }^{23}$, son las mujeres quienes reportan en mayor porcentaje una relación perjudicial con bebedores problemáticos. La evidencia en otros países, como Estados Unidos de Norteamérica ${ }^{21}$ presenta tasas similares en situaciones como los accidentes de tránsito y el daño a la propiedad del individuo.

La principal limitación de este estudio es no ser representativo de la población general chilena. Además de un posible sub-registro, dado que en ocasiones la encuesta era realizada con la presencia del bebedor problema en el hogar: en otros estudios similares la subdeclaración es baja por lo que se espera que en Chile sea similar. Cabe señalar que el muestreo por área geográfica empleado por acuerdo de todos los países del estudio colaborativo, exigía una división intencionada por regiones, que en el caso chileno llevó a dividir al país, de acuerdo al protocolo internacional en cinco regiones (Norte, Centro, Sur y Área Metropolitana). Este mismo protocolo se centra en encuestar sólo a mayores de 18 años y menores de 65. El hecho de que esta muestra presente un mayor porcentaje de habitantes correspondientes a conglomerados urbanos de más de 50.000 habitantes, influye en el mayor número de casos con estudios secundarios y universitarios, que los existentes en la población general. El contar con una muestra de mayor nivel educacional hace que aumente el número de encuestados sin afiliación religiosa, situación que en toda Hispano-américa se liga a mayor escolaridad ${ }^{24}$. Asimismo, tanto el diseño como la duración de la encuesta requiere un cierto nivel cognitivo para responderla en su totalidad, lo que implicó excluir del análisis final a $28,2 \%$ de encuestados que no cumplieron con este criterio.

\section{Conclusiones y perspectivas}

Estos hallazgos confirman que el daño colateral por consumo de alcohol, es un tema relevante dada su frecuencia en la población general. Las mujeres y las dueñas de casa se ven más afectadas, apareciendo como un foco a intervenir para evitar las situaciones más graves y disminuir los altos porcentajes de daño encontrados.

En general, el alcohol ha sido estudiado como un factor de riesgo para diversos problemas. Los estudios se han focalizado en el consumidor, más que en su entorno. Es necesario profundizar en los costos personales, materiales y financieros con estudios como este. El estudio colaborativo internacional en que Chile participa hará posible entregar evidencia para una mejor comprensión de las consecuencias del consumo de alcohol, así como del daño infligido a quienes rodean al consumidor. La evidencia informada en este estudio será útil para los tomadores de decisiones. Posteriores análisis de esta misma base de datos pueden orientar con respecto a las mejores intervenciones preventivas para disminuir el impacto negativo del consumo de alcohol.

\section{Referencias}

1. World Health Organization. 61 World Health Assembly. Order of the Day 11.10. Strategies to reduce the harmful use of alcohol: draft global strategy. Secretariat Report. Geneva: WHO; 2008. Disponible en: http://apps.who. int/gb/ebwha/pdf_files/WHA63/A63_13-en.pdf [Consultado el 25 de junio de 2015].

2. Laslett AM, Catalano P, Chikritz T, et al. The Range and magnitude of Alcohol's Harm to Others. Canada: Faer Foundation; 2012.

3. Ministerio de Salud; Comité Interministerial. Estrategia Nacional sobre Alcohol: Reducción del consumo de riesgo y sus consecuencias sociales y sanitarias. Propuesta de un desafío bicentenario para Chile. Santiago de Chile. Enero 2010. Disponible en: http://web.minsal.cl/ 
sites/default/files/files/2\%20Estrategia $\% 20$ Nacional $\% 20$ sobre\%20Alcohol_b.pdf [Consultado el 25/6/2015].

4. Rehm J, Room R, Monteiro M, Gmel G, Graham K, et al. Comparative quantification of health risks: global and regional burden of disease due to selected risk factors. Geneva: OMS; 2004: 959-1008. Disponible en: http://apps.who.int/iris/. [Consultado el 25 de junio de 2015].

5. Ministerio de Salud. Segundo Estudio de Carga de Enfermedad y Carga Atribuible. Chile: Departamento de Epidemiología Universidad Católica de Chile; 2007. Disponible en: http://epi.minsal.cl/epi/html/invest/ cargaenf2008/informe\%20final\%20carga_Enf_2007.pdf. [Consultado el 25 de junio de 2015].

6. World Health Organization. Global Status Report on Alcohol and Health. Geneva: WHO; 2011. Disponible en: http://www.who.int/substance_abuse/publications/ global_alcohol_report/msbgsruprofiles.pdf . [Consultado el 25 de junio de 2015.]

7. Vial A, Fernández, S. El costo económico del alcoholismo. Algunos marcadores para Chile. Documento de investigación No 31. Documento de trabajo publicado por el Departamento de Economía, Universidad de Chile. Santiago, 1979.

8. Ministerio de Salud. Impacto Económico del uso de alcohol en Chile. Santiago de Chile: Ministerio de Salud, Gobierno de Chile; 2010.

9. Comisión Nacional de Seguridad de Transito. Informe sobre Análisis Espacial de Locales de Expendio de Alcohol y Accidentes de Tránsito relacionados con Alcohol, en la Región Metropolitana. Diagnóstico 2011. Santiago de Chile: CONACET; 2012. Disponible en http://www. conaset.cl/wp-content/uploads/2013/11/accidentes_alcohol_locales_de_consumo_2011.pdf [Consultado el 25 de junio de 2015.]

10. Babor T, Caetano R, Casswell S, Edwards G, Giesbrecht N, Graham K, et al. Alcohol: no ordinary commodity: research and public policy. Oxford: Oxford University Press; 2003.

11. Larraín S, Bascuñán C. Maltrato Infantil y Relaciones Familiares en Chile: Análisis Comparativo. 1998-2002-2006. Santiago de Chile: UNICEF; 2006. Disponible en: http://www.scielo.cl/scielo.php?pi$\mathrm{d}=$ S0370-41062008000700011\&script=sci_arttext [Consultado el 25 de junio de 2015].

12. Marty C, Carvajal C. Maltrato Infantil como factor de riesgo de trastorno por estrés postraumático en la adultez. Rev Chil Neuro-Psiq. 2005; 43 (3):180-187. Disponible en: http://www.scielo.cl/scielo.php?script=sci_arttext\&pid=S0717-92272005000300002 [Consultado el 25 de junio de 2015]
13. Dube S, Anda R, Felitti V, Chapman D, Williamson D, Giles W. Childhood Abuse, Household Dysfunction, and the Risk of Attempted Suicide Throughout the Life Span. Findings From the Adverse Childhood Experiences Study. JAMA 2001; 286(24): 3089-96. Disponible en: http://jama.jamanetwork.com/article.aspx?articleid=194504 [Consultado el 25 de junio de 2015].

14. Florenzano R, Hernández C, Venegas L, Larraín L, Godoy J, Sieverson C. Alcoholismo en la familia y salud mental en hijos adultos. Revista Chilena Neuropsiquiatría. 2013; 51:66. Disponible en: http://revistagpu. cl/2014/GPU_marzo_2014_PDF/INV_Alcoholismo.pdf [Consultado el 25 de junio de 2015].

15. Castillo-Carniglia A, Pizarro E, Luengo D, Soto-Brandt G. Consumo de alcohol y autoinforme de eventos violentos en Chile. Adicciones 2014; 46 (1): 46-53. Disponible en: http://www.adicciones.es/files/Castillo_ARTIC. pdf [Consultado el 25 de junio de 2015].

16. Ansoleaga E, Castillo-Carniglia A. Associations between social vulnerability, employment conditions and hazardous alcohol consumption in Chile. Drug and Alcohol Review 2013; 32 (3): 254-61. Disponible en: http://onlinelibrary.wiley.com/doi/10.1111/j.14653362.2012.00522.x/abstract [Consultado el 25 de junio de 2015].

17. WHO/Thai Health International Collaborative Research Project. The Harm to Others from Drinking: Master Protocol. Thailand. Documento publicado por Thai Health Promotion Foundation; 2012.

18. Instituto Nacional de Estadísticas. Compendio Estadístico 2013. Santiago de Chile: Instituto Nacional de Estadísticas; 2013. Disponible en: http://www.ine.cl/canales/ menu/publicaciones/calendario_de_publicaciones/pdf/ COMPENDIO_2013.pdf. [Consultado el 25 de junio de 2015].

19. World Health Organization. Process of translation and adaptation of instruments. http://www.who.int/substance_abuse/research_tools/translation/en/. Acceso March [Consultado el 28 de marzo de 2015].

20. Greenfield T, Ye Y, Kerr W, Bond J, Rehm J, Giesbrecht N. Externalities from Alcohol Consumption in the 2005 US National Alcohol Survey: Implications for Policy. International Journal of Environmental Research and Public Health. 2009; 6, 3205-3224. Disponible en: http:// www.ncbi.nlm.nih.gov/pmc/articles/PMC2800345/. [Consultado el 25 de junio de 2015].

21. Connor J, You R, Casswell S. Alcohol-related harm to others: a survey of physical and sexual assault in New Zealand. The New Zealand Medical Journal 2009; 122: 1-8.

22. Laslett AM, Catalano P, Chikritzhs T, Dale C, Doran 
C, Ferris J, et al. The range and magnitude of alcohol's harm to others. Fitzroy, Australia: Turning Point Alcohol \& Drug Centre; 2010. Disponible en: https://www. melbourneinstitute.com/downloads/hilda/Bibliography/ Other_Publications/pre2010/Laslett_etal_Alcohol's_ Harm_to_Others.pdf [Consultado el 25 de junio de 2015].
23. Pew Research Center. The Future of World Religions: Population Growth Projections. 2010-2050. 2015. Why Muslims Are Rising Fastest and the Unaffiliated Are Shrinking as a Share of the World's Population. Disponible en: http://www.pewforum.org/files/2015/03/ PF_15.04.02_ProjectionsFullReport.pdf [Consultado el 25 de junio de 2015]. 